\title{
Pheromone-based Dynamic Coordination for Manufacturing System
}

\author{
Lei Wang ${ }^{*}$, Ming Li, Jingcao Cai, Zhihu Liu and Zhen Li
}

School of Mechanical and Automotive Engineering, Anhui Polytechnic University, Wuhu, 241000, China

\begin{abstract}
Manufacturing enterprises must face increasingly difficult production environments. External factors such as varying product variants and varying quantities as well as internal factors such as reworking and resource breakdowns pose a high challenge for task allocation and control. Inspired by the similarity between ant foraging behavior model and task allocation in manufacturing system, a pheromone-based coordination mechanism for manufacturing system is proposed. The pheromone-based static and dynamic coordination algorithms for task allocation are given. Experimental results demonstrate that not only the relatively optimal processing costs but also balanced use of machines can be achieved by using the proposed algorithm, and that it has high adaptability to the disturbance from inside or outside of manufacturing system. Therefore, it provides a feasible new idea for dealing with an actual allocation problem of production tasks for manufacturing system.
\end{abstract}

Keywords: Dynamic coordination, manufacturing system, pheromone, task allocation.

\section{INTRODUCTION}

The environment within which manufacturing systems operate is characterized by rapid change than ever before. The unforeseen disturbances include: the arrival of new orders, order cancellations, changes in order priority, processing delays, changes in release dates, machine breakdowns, etc. $[1,2]$. Hence, such dynamic manufacturing systems require dynamic coordination and control to make full use of system resources, and then improve the system performance. The traditional manufacturing control architectures are not designed to exhibit good agility and adaptation. In fact, their centralized and hierarchical control approaches present good production optimization, but the rigidity and centralization of the control structure implies a weak response to disturbances, therefore, the substitutional heterarchical control architecture has been proposed and investigated $[3,4]$. On the other hand, heterarchical manufacturing control architecture presents a good response to change and unpredictable disturbances, but as autonomous manufacturing cells have different information, knowledge, goals and understanding of current situations, and decisions are based on partial knowledge of the system, their action plan may be disparate or even conflictive. Therefore the global production optimization is not guaranteed $[5,6]$. Thus an appropriate approach is necessary to coordinate and integrate the behavior of manufacturing system.

Considering the already developed ant colony optimization (ACO) methodology [7], ACO has a powerful capacity to find out good solutions to combinatorial optimization problems, Therefore, ACO has been widely applied to solving various combinatorial optimization problems such as traveling salesman problem (TSP) [8], job-shop scheduling problem (JSP) [9], task allocation problem [10], etc. This paper uses an indirect coordination approach based on pheromone to optimize global performance for manufacturing system.

The remainder of this paper is organized as follows. The pheromone-based coordination mechanism is given in section 2. The pheromone-based static and dynamic coordination algorithms are proposed in section 3. An example is presented to demonstrate the validity of this approach to control task allocation in section 4. Finally, some concluding remarks are given in section 5 .

\section{PHEROMONE-BASED COORDINATION MECH- ANISM}

Pheromone-based coordination mechanism is inspired by the foraging behavior of real ants. Real ants are capable of finding the shortest path from a food source to their nest without using any direction communication. Instead, they communicate information about the food source via depositing a chemical substance, called pheromone, on the paths. The following ants are attracted by the pheromone. Since the shorter paths have higher traffic densities, these paths can accumulate higher proportion of pheromone. Hence, the probability of ants following these shorter paths would be higher than that of those following the longer ones.

Much to our surprise, just using this simple strategy, an ant colony can find the optimal route from nest to food source quickly in a very complex environment (in contrast with their intelligence) [11]. 


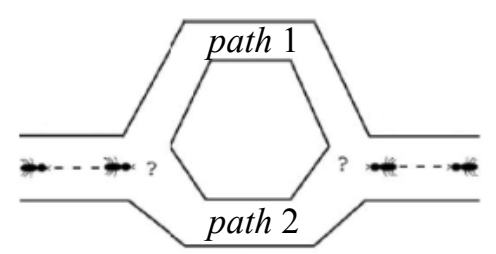

(a)

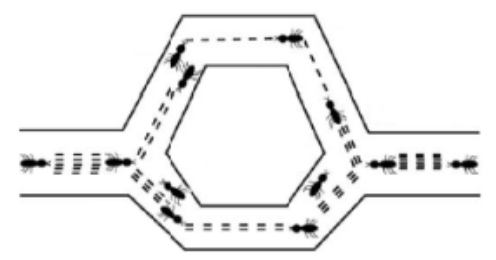

(c)

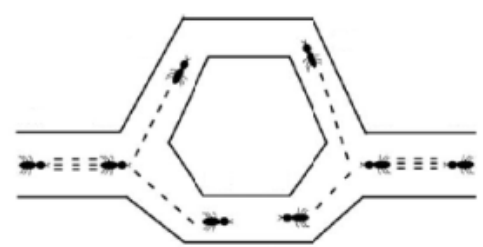

(b)

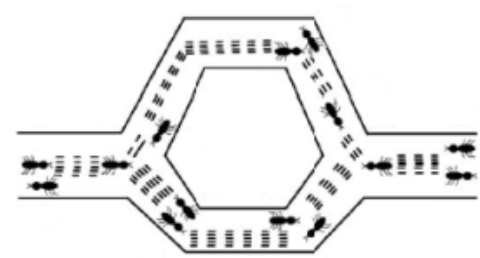

(d)

(a) Ants arrive at a decision point. (b) Some ants choose the upper path and some the lower path. The choice is random. (c) Since ants move at approximately constant speed, the ants which choose the lower, shorter, path reach the opposite decision point faster than those which choose the upper, longer, path. (d ) Pheromone accumulates at a higher rate on the shorter path. The number of dashed lines is approximately proportional to the amount of pheromone deposited by ants.

Fig. (1). The process for real ants to find a shortest path [9].

Fig. (1) can explain why an ant colony can achieve such good performance [9]. When an ant is searching for the nearest food source and comes across with several possible paths (path 1 and path 2), it tends to choose the path with the largest concentration of pheromone, with a certain probability. After choosing the path, it deposits a certain quantity of pheromone, increasing the concentration of pheromones in this path. The ants return to the nest using always the same path, depositing other portion of pheromone on the way back. Imagine then, that two ants at the same location choose two different paths at the same time. The pheromone concentration on the shortest way (path 2) will increase faster than the other (path 1): the ant that chooses this way, will deposit more pheromones in a smaller period, because it returns earlier. If a whole colony of thousands of ants follows this behavior, soon the concentration of pheromone in the shortest path will be much higher than the concentration in other paths. Then the probability of choosing any other way will be very small and only very few ants among the colony will fail to follow the shortest path. There is another phenomenon related with the pheromone concentration. Since it is a chemical substance, it tends to evaporate in the air, so the concentration of pheromones vanishes along the time. In this way, the concentration of the less used paths will be much lower than that on the most used ones, not only because the concentration increases in the other paths, but also because their own concentration decreases with time [9].

A further research indicates [12] that the behavior model for ant colony to choose path for food can be presented in (1).

$$
p_{i}=\frac{\left(k+c_{i}\right)^{n}}{\sum_{j=1}^{m}\left(k+c_{j}\right)^{n}}
$$

Where, $p_{i}$ is the probability for ant to choose path $i$ for food; $c_{i}$ and $c_{j}$ are the quantity of pheromone on path $i$ and $j$, respectively; $k$ is attractive degree for no pheromone paths; $n$ is a nonlinear coefficient, and we set $n$ to 1 in this paper.

\section{PHEROMONE-BASED DYNAMIC COORDINA- TION}

\subsection{Pheromone-based Static Coordination Algorithms}

In an ants' food foraging process, pheromone is the basic information element and resides in the route it associates. The function of pheromone is to indicate the attraction of its resident route. The prominent characteristic of pheromone is simplicity [12]. Therefore, the task allocation can be looked as ants' food foraging process. Generally speaking, a task can be finished by several routes; in order to make each route have a chance to be selected, so at the beginning, we set an initial value for each route that has the ability to finish the given task, as shown in (2).

$$
\rho[i][j]=\left\{\begin{array}{l}
c_{0} \text { if work piece jcan be finished by } \\
0 \text { route iotherwise }
\end{array}\right.
$$


When a task needs to select a route to process their work pieces, the work piece firstly perceives the pheromone quantum of each feasible route and selects a process route by route's selecting probability, which can be obtained from (1), as shown in (3).

$$
p(i)=\frac{\rho[i][j]}{\sum_{i=1}^{m} \rho[i][j]}
$$

Where, $i$ represents one process route and $j$ represents the work piece of the task; $p(i)$ is the selecting probability of process route $i$ and $\rho[i][j]$ is route $i$ 's pheromone to work piece $j$.

After that the selecting algorithm generates a random number and chooses a process route for processing the work piece $j$ according to calculated selecting probability. Then the selected route will get the award, and the "effective pheromone" would be increased. Meanwhile, the involving machine's available processing time on the selected process route will gradually be reduced. Therefore, a pheromone penalty function $P(t)(0<P(t)<A(c)$ are used to reduce the strength of the pheromone in order to reduce the probability of its being selected again, as shown in (4).

$$
\rho[i][j]=\rho[i][j]+A(c)-P(t)
$$

Where, $c$ is the total processing cost of the selected route; $A(c)$ is the increased pheromone quantum, and the increment is inversely proportional to the processing cost. $P(t)$ is a decreasing function on the least available time machine of the process route.

When the available time of a certain machine of the process route is less than the corresponding process time processed by this machine for the next work piece, then the pheromone value of the process route automatically becomes zero. Particularly, when a machine's available time is zero, the pheromone value is set to zero for all process routes associated with the machine. The flow chart of pheromonebased coordination algorithm for task allocation is shown in Fig. (2).

\subsection{Pheromone-based Dynamic Coordination}

There are many sources of uncertainty in a real-world manufacturing system, which trigger disturbance events in dynamic adaptation manufacturing system control. Generally speaking, there are two types of disturbances, which are resource-related disturbance and source-related disturbance $[1,6]$.

1) Resource-related disturbance refers to the disturbance caused by unreliability coming from resources (machines) in the shop floor, including machine recovery and machine breakdown.
2) Source-related disturbance refers to the disturbance caused by the changes in orders, including existing order/job cancellation and new order/job arrival.

The resource-related disturbance is considered in this paper.

Fig. (3) shows the progress of dynamic coordination by using pheromone-like technique when a machine has a malfunction at time $t_{0}$. And the specific dynamic coordination mechanism works as follows:

(1) Set the pheromone value to zero for the malfunction machine.

(2) Find all machines which have the ability to replace the malfunction machine, and calculate pheromone values $S_{i}$ and the remaining available time $T_{i}$ for these alternative machines at the current moment.

(3) Calculate the operating pheromone value for each alternative machine, as shown in (5).

$Q_{i}=\lambda_{1} S_{i}+\lambda_{2} T_{i}$

Where, $\lambda_{1}$ and $\lambda_{2}$ are the cost weight and load weight of the machine $\left(\lambda_{1}+\lambda_{2}=1\right)$ respectively.

(4) Transferred the unprocessed work pieces on the malfunction machine to an alternative machine according to task allocation algorithm based on pheromone. The selected probability for each alternative machine is as follows.

$p_{i}=\frac{Q_{i}}{\sum Q_{i}}$

(5) The selected machines and the old machines form a new dynamic manufacturing dell.

(6) After the task is completed, the dynamic manufacturing cell will automatically disappear.

\section{EXAMPLE AND RESULT}

There are three tasks that need to be processed, and there are 9 machines in a manufacturing plant. The production information for these tasks is shown in Table $\mathbf{1}$.

Algorithmic parameters are selected as follows: $c_{0}=100$, $\alpha(c)=10000 / c, P\left(\alpha\left(t_{\text {min }}\right), A(c)\right)=\alpha\left(t_{\text {min }}\right) * \alpha(c)$, where $\alpha\left(t_{\text {min }}\right.$ )$=D /\left(D+9 t_{\min }\right), D=1500$.

Solved by pheromone-based algorithm, the following results are obtained: $n_{11}=25 ; n_{12}=28 ; n_{13}=27 ; n_{21}=19 ; n_{22}=30$; $n_{23}=41 ; n_{31}=26 ; n_{32}=22 ; n_{33}=22 . n_{i j}=N(i=1,2,3 ; \mathrm{j}=1,2,3)$ denotes that $N$ work pieces of $P_{i}$ will be processed on the route of $P_{i}-R_{j}$ ( $N$ is less than or equal to the batch size of $\mathrm{P}_{i}$ ). For example, $n_{11}=25$ denotes that eighteen work pieces of $P_{1}$ are planned to be processed on the $P_{1}-R_{1}$ route. 


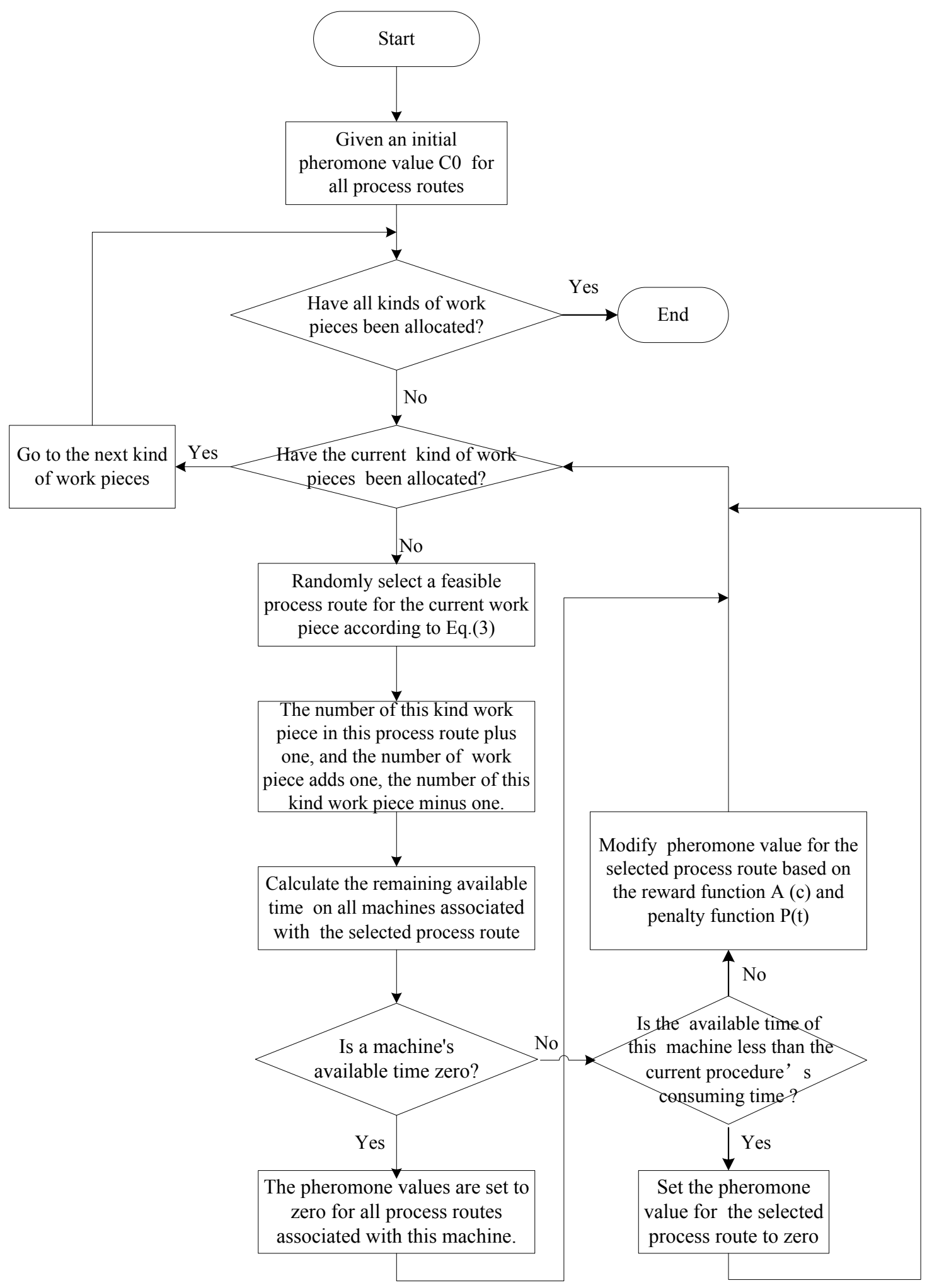

Fig. (2). Pheromone-based static coordination process. 


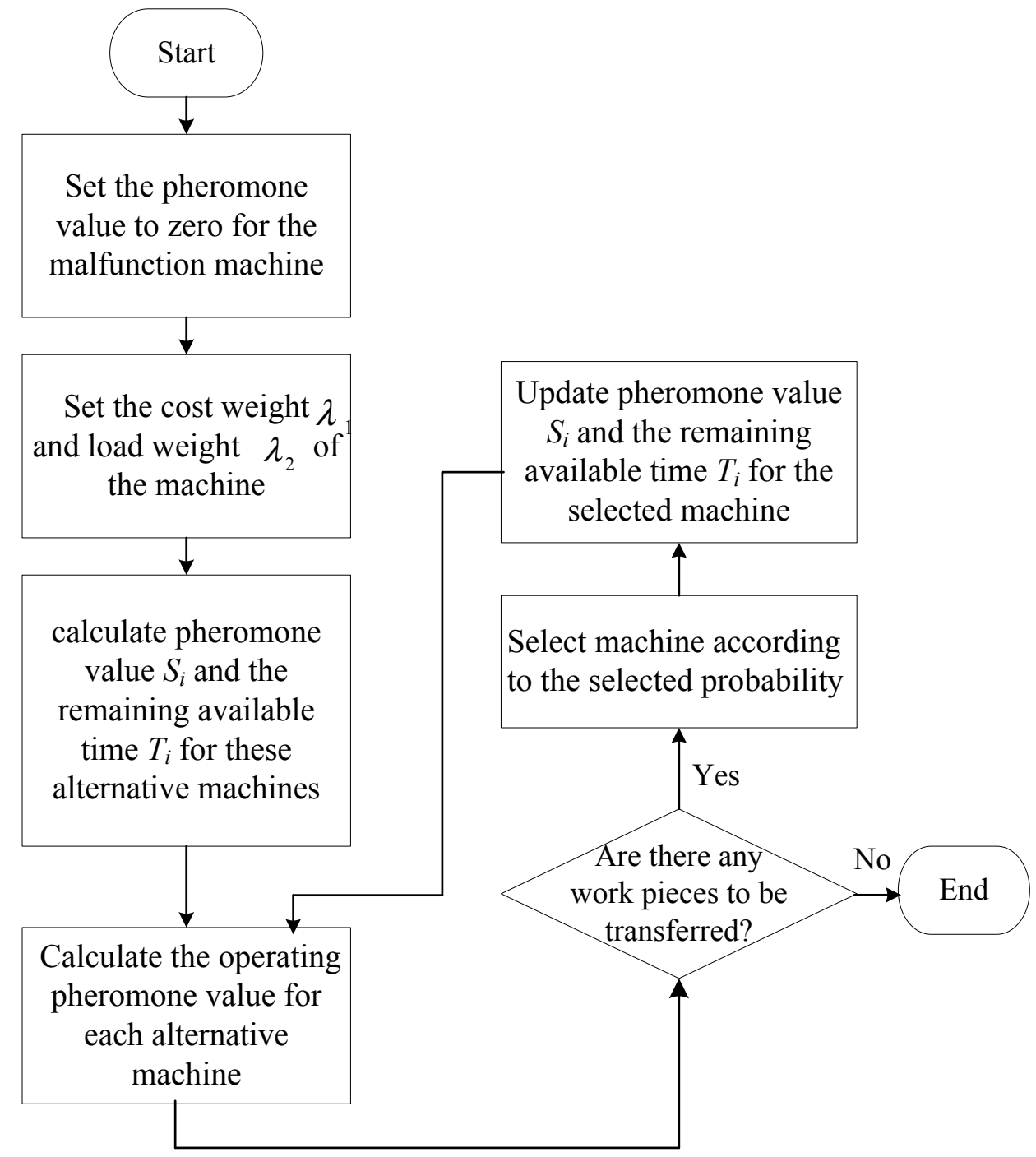

Fig. (3). Pheromone-based dynamic coordination.

Table 1. Production information for tasks.

\begin{tabular}{|c|c|c|c|c|c|c|}
\hline Task & Quantity & Routes & \multicolumn{4}{|c|}{ Machine/Processing Time /Cost Per Time Unit } \\
\hline \multirow{3}{*}{$\mathrm{P}_{1}$} & \multirow{3}{*}{80} & $1-1$ & $1 / 12 / 4$ & $3 / 18 / 3$ & $4 / 15 / 2$ & $6 / 15 / 8$ \\
\hline & & $1-2$ & $2 / 12 / 5$ & $5 / 10 / 5$ & & \\
\hline & & $1-3$ & $8 / 15 / 6$ & $2 / 12 / 5$ & & \\
\hline \multirow{3}{*}{$\mathrm{P}_{2}$} & \multirow{3}{*}{90} & $2-1$ & $2 / 10 / 5$ & $5 / 5 / 5$ & $8 / 10 / 6$ & $1 / 6 / 4$ \\
\hline & & $2-2$ & $4 / 8 / 2$ & $3 / 15 / 3$ & $9 / 10 / 2$ & $7 / 15 / 12$ \\
\hline & & $2-3$ & $1 / 8 / 4$ & $4 / 8 / 2$ & $6 / 5 / 8$ & \\
\hline \multirow{3}{*}{$\mathrm{P}_{3}$} & \multirow{3}{*}{70} & $3-1$ & $8 / 6 / 6$ & $9 / 10 / 2$ & $7 / 25 / 12$ & \\
\hline & & $3-2$ & $6 / 12 / 8$ & $1 / 10 / 4$ & $3 / 5 / 3$ & \\
\hline & & $3-3$ & $5 / 10 / 5$ & $2 / 8 / 5$ & $8 / 15 / 6$ & \\
\hline
\end{tabular}


Table 2. Pheromone quantum on each machine.

\begin{tabular}{|c|c|c|}
\hline Machine ID & Machine Type & Released Pheromone \\
\hline \hline M1 & lathe & 829 \\
\hline M2 & milling machine & 536 \\
\hline M3 & machining center & 811 \\
\hline M4 & grinding machine & 695 \\
\hline M5 & lathe & 837 \\
\hline M6 & planning machine & 315 \\
\hline M7 & boring machine & 758 \\
\hline M8 & grinding machine & 141 \\
\hline M9 & drilling machine & \\
\hline
\end{tabular}

Table 3. Task allocation results for remaining $\mathbf{3 0}$ work pieces.

\begin{tabular}{|c|c|c|}
\hline Machine ID & Machine Type & Allocated Number \\
\hline \hline M3 & machining center & 19 \\
\hline M7 & boring machine & 11 \\
\hline
\end{tabular}

The pheromone quantum on each machine is shown in Table 2.

Assume that M9 is failure at time $t_{0}$, and then the remaining 30 work pieces can't be processed on M9. The alternative M3 and M7 can processed them. Set the cost weight $\lambda_{1}$ to 0.6 and load weight $\lambda_{2}$ to 0.4 for M3 and M7 respectively.

According to the dynamic coordination of the above steps, the obtained allocated work pieces on each alternative machine M3 and M7, as shown in Table 3.

The tasks can be well allocated between different alternative machines by adjusting the weight factors $\lambda_{1}$ and $\lambda_{2}$. It not only ensure that the relatively low-cost and alternative machines can be well selected to process the remaining work pieces, but also take into account the full use of the idle machines' processing capabilities. Therefore, the manufacturing system can be fully utilized as soon as possible. A new virtual dynamic manufacturing cell can be formed by adding the new machines to the original manufacturing cell. Therefore, some tasks can be well allocated by coordinating among several machines or manufacturing cells, which can learn from each other, in order to optimize the system's overall performance.

\section{CONCLUSION}

Inspired by social insects, a new pheromone-based dynamic coordination for manufacturing system is presented in this paper. According to that, a pheromone-based task allocation algorithm is proposed. Based on pheromone technique, an example is given and experimental results confirm that the proposed approach is feasible for dynamic coordination problems.

Pheromone-based dynamic coordination approach has following characteristics: (1) Tasks allocation and resources optimization can be combined effectively, and (2) The approach is not only simple, but also feasible.

The application of pheromone-based coordination approach in manufacturing system is focused on basic task allocation problems and is only tested by using computer, therefore, it is still preliminary and further research work is needed. Future work will consider the following issues: (1) applying the proposed coordination approach to real industrial cases, (2) further exploring ant agent coordination for dynamic re-scheduling coordination control which can provide a schedule immediately and efficiently, and (3) improving the proposed coordination algorithms so as to improve allocation efficiency. 


\section{CONFLICT OF INTEREST}

The authors confirm that this article content has no conflict of interest.

\section{ACKNOWLEDGEMENTS}

This paper is supported by the National Natural Science Foundation of China (Grant No. 51305001), Natural Science Foundation for Youths of Anhui Province (Grant No. 1208085QE94, 1308085ME78, 1308085ME65), Anhui Engineering Technology Research Center of Automobile New Technology, and Universities Natural Science Research Project of Anhui Province (Grant No. KJ2013B033, TSKJ2014B12). The authors would like to thank the referees, the editors and the anonymous reviewers for their helpful comments and constructive suggestions.

\section{REFERENCES}

[1] W. Xiang, and H.P. Lee, "Ant colony intelligence in multi-agent dynamic manufacturing scheduling", En. Appl. Artif. Intell., vol. 21, pp. 73-85, 2008.

[2] N.G. Hall, and C.N. Potts, "Rescheduling for new orders", Oper. Res., vol. 52, no. 3, pp.440-453, 2004.
[3] D.M. Dilts, N.P. Boyd, and H.H. Whorms, "The evolution of control architectures for automated manufacturing systems", J. Manuf. Syst., vol. 10, no. 1, pp. 79-93, 1991

[4] N.A. Duffie, and R.S. Piper, "Non-hierarchical control of a flexible manufacturing cell", Robot Comput. Integr. Manuf. Syst., vol. 3, no. 2, pp. 175-179, 1987

[5] P. Leitao, and F. Restivo, "ADACOR: a holonic architecture for agile and adaptive manufacturing control", Comput. Indust., vol. 57, pp. 121-130, 2006.

[6] L. Wang, D.B. Tang, W.B. Gu, K. Zheng, W.D. Yuan, and D.S. Tang, "Pheromone-based coordination for manufacturing system control", J. Intel. Manuf., vol. 23, no. 3, pp. 747-757, 2012.

[7] C.M. Dorigo, V. Maniezzo, and A. Colorni, "The ant system: Optimization by a colony of cooperating agents", IEEE Trans. Syst., Man, Cybern. Part B, vol. 26, pp. 29-41, 1996.

[8] Z.C.S.S. Hlaing and M.A. Khine, "Solving traveling salesman problem by using improved ant Colony optimization algorithm", Int. J. Inform. Educ. Technol., vol. 1, no. 5, pp. 404-409, 2011.

[9] S. Kumar, and C.S.P. Rao. "Application of ant colony, genetic algorithm and data mining-based techniques for scheduling", Robotics Comput. Integr. Manuf., vol. 25, pp. 901-908, 2009.

[10] S.H. Liu, Y. Zhang, "Multi-robot task allocation based on particle swarm and ant colony optimal," J. Northeast Normal Univ., vol. 41, no. 4, pp. 68-72, 2009.

[11] S. Camazine, F.J.-L. Deneubourg, N.R. Franks, J. Sneyd, G. Theraulaz, and E. Bonabeau., Self-organization in biological systems, Princeton: Princeton University Press, 2001.

[12] Q.L. Gao, X. Luo, and S.Z. Yang, "Stigmergic cooperation mechanism for shop floor control system", Int. J. Adv. Manuf. Technol., vol. 25, pp. 743-753, 2005.

Received: September 16, 2014

Revised: December 23, 2014

Accepted: December 31, 2014

(c) Wang et al.; Licensee Bentham Open.

This is an open access article licensed under the terms of the Creative Commons Attribution Non-Commercial License (http://creativecommons.org/licenses/by-nc/3.0/) which permits unrestricted, non-commercial use, distribution and reproduction in any medium, provided the work is properly cited. 